\title{
Finite Element analysis of the combined effect of strain hardening and friction in elementary processes of forging
}

\author{
A.M. Camacho ${ }^{1, a}$, M.M. Marín ${ }^{1, b}$, L. Sevilla ${ }^{2, c}$ and C. Bernal ${ }^{1, d}$ \\ ${ }^{1}$ Department of Manufacturing Engineering, National Distance University of Spain (UNED), Madrid, \\ Spain \\ ${ }^{2}$ Department of Manufacturing Engineering, University of Malaga, Malaga, Spain \\ aamcamacho@ind.uned.es, 'bmmarin@ind.uned.es, Isevilla@uma.es, 'cbernal@ind.uned.es
}

Keywords: metal forming, elementary forging processes, strain hardening, friction, Finite Element Method.

\begin{abstract}
Forging processes have been studied since years. However, recently these studies are gaining in importance because of the increasing emergence of non conventional forging processes such as LIF processes, in order to improve their efficiency and to fit the production requirements. In this work elementary forging processes are studied under plane strain conditions in order to evaluate the combined effect of strain hardening and friction in forces and contact pressure distributions by a FE analysis. For this purpose, different base to height ratios $(b / h)$ of the workpiece have been considered, with different friction conditions. All cases have been solved for both a rigid perfectly plastic material and a strain hardened one. It is observed that the effect of the strain hardening on the forces and contact pressures is higher when the friction conditions become more extreme. The results do not depend on the base to height ratios when frictionless conditions are assumed.
\end{abstract}

\section{Introduction}

Metal forming industry is interested in improving the quality of final products, increasing the productivity and achieving maximum efficiency at minimum cost, consistent with the required quality standards. An overall knowledge of the processes and an exhaustive analysis of them are required in order to reach these aims. Forging processes are widely used for producing parts of excellent mechanical properties with minimum waste of material. The wokpiece is plastically deformed in one or more operations where very large plastic deformations occur. Open-die forging, and concretely upsetting, is a forging process that consists of reducing the height, $h$, of a workpiece between two flat dies (also called plattens) by means of compressive forces. Because constancy of volume is maintained, the reduction in height increases the base, $b$, of the forged part. In the last decades investigations have been focused in the development of new processes [1], such as Localized-Incremental Forging (LIF) processes [2], which can overcome some of the limitations and/or disadvantages of conventional ones. However, to improve the knowledge in these new processes, it is advisable to get detail information about the conventional ones and to develop robust finite element models. In the first 70's, the Finite Element Method (FEM) was established an indispensable tool for metal forming analysis. This numerical technique allows studying complex geometries and boundary conditions and quite realistic material responses [3]. The Finite Element Method is a powerful method that takes into account the three main contributions to the total energy (homogeneous deformation, friction and distortion). Conventional analytical methods such as the Upper Bound Theorem [4] can also be successfully applied to analyze this kind of problems. However, FEM is specially recommended when phenomena such as the strain hardening want to be incorporated and/or stress and strain distributions are required. Using FEM it is possible to study at the same time the influence of several technological factors [5], especially those ones like strain hardening that usually cannot be easily introduced in analytical conventional methods. In this work, FE analysis is used to evaluate the combined effect of strain hardening and friction in workpieces with different geometries under plane strain conditions. 


\section{Methodology}

Many factors can affect the required energy in this process. Some of the most important variables are the friction [6] and the strain hardening of the material. Therefore, in order to get accurate results, the FE model should take into account the combination of both phenomena. This work shows that a third element has to be considered as well: the geometry of the workpiece. For this purpose, different base to height $(b / h)$ ratios have been chosen, being $h=1$. The reduction in height is defined as $r(\%)=$ $100 \cdot\left(h_{i}-h_{f}\right) / h_{i}$. A sliding friction model is used by considering the Coulomb friction coefficient, $\mu$.

The numerical simulation is used to predict the forging load under several conditions of friction between the platens and the workpiece. Forces are expressed by means of the dimensionless ratio $F /\left(A_{i} S\right)$, where $A_{i}$ is the initial contact area, and $S=2 k$ is the yield stress under plane strain conditions. Also, contact pressures at the die are going to be evaluated. Contact pressures are obtained by the output variable CPRESS. In order to evaluate the effect of strain hardening, the material of the workpiece has been modeled considering two material behaviors in the plastic region: a Rigid Perfectly Plastic-RPP material and a Strain Hardened-SH one, whose main properties are gathered in Table 1.

Table 1. Material properties for two types of material

\begin{tabular}{|c|c|c|c|}
\hline & $\begin{array}{c}\text { Rigid Perfectly Plastic } \\
\text { (RPP) }\end{array}$ & \multicolumn{2}{|c|}{$\begin{array}{c}\text { Strain Hardened } \\
\text { (SH) }\end{array}$} \\
\hline Flow Stress (GPa) & 0.7 & 0.7 & 3.7 \\
\hline Plastic Strain & 0.0 & 0.0 & 10.0 \\
\hline
\end{tabular}

Both forging load and contact pressure distributions are calculated assuming a rigid perfectly plastic material and additionally, a strain hardened one. The FEM code used is ABAQUS/Standard [7] that it is a general purpose code of implicit methodology. The workpiece has been meshed by means of the CPE4R element type. It is a continuum, plane strain, linear interpolation and reduced integration element.

\section{Results}

Forces analysis. Fig. 1 presents the obtained forces for different friction coefficients and a reduction of $50 \%$. This figure compares the results for a rigid perfectly plastic material and for a strain hardened one, and two different geometries of the workpiece have been also considered.

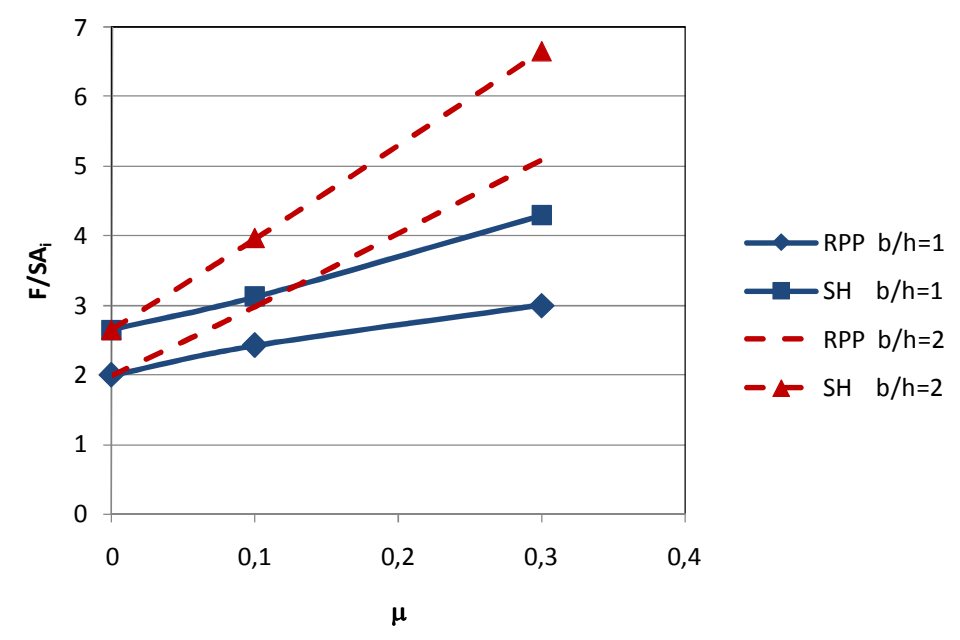

Fig. 1. Forces analysis considering two material models (Rigid Perfectly Plastic-RPP and Strain Hardened-SH), two base to height ratios and different friction conditions $(r=50 \%)$ 
As expected, forces are higher when considering a SH material than a RPP one. As a general trend, the higher the friction, the higher the required forces. It can also be observed that the initial geometry $(b / h)$ of the workpiece has a strong influence on the results when friction is considered; however, forces do not depend on the $b / h$ ratio when frictionless conditions are involved. Another interesting result is that, for the same $b / h$ ratio, the effect of the strain hardening on the forces is higher when the friction conditions become more extreme. This can be clearly checked because the two lines belonging to each $\mathrm{b} / \mathrm{h}$ ratio do not have a parallel evolution.

Contact pressures analysis. Contact pressure distributions for both kinds of materials are observed in Fig. $2 a$ and $2 b$ without and with friction, respectively. In the first case (Fig. 2a), contact pressures do not seem to depend on the $b / h$ ratio (as it happened with forces). The main differences are noted when the material undergoes strain hardening; in this case, the higher the strain hardening, the higher the contact pressures.

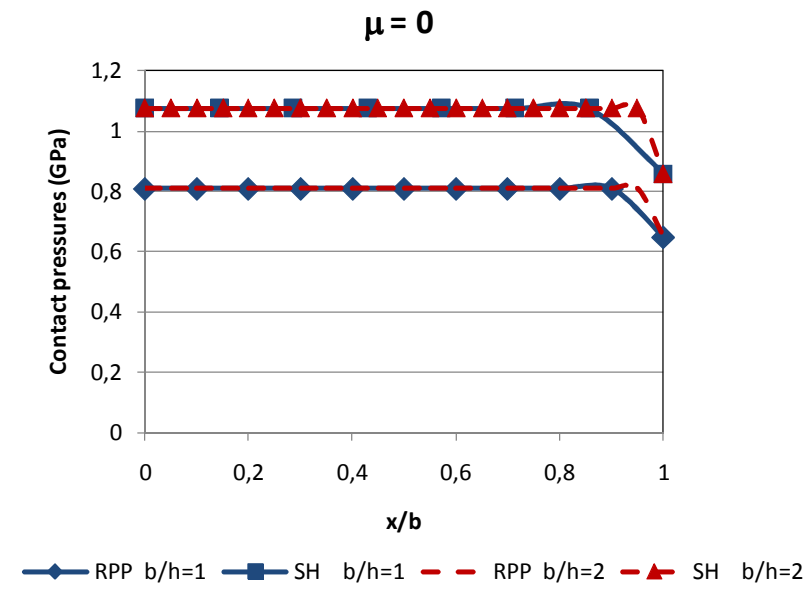

a)

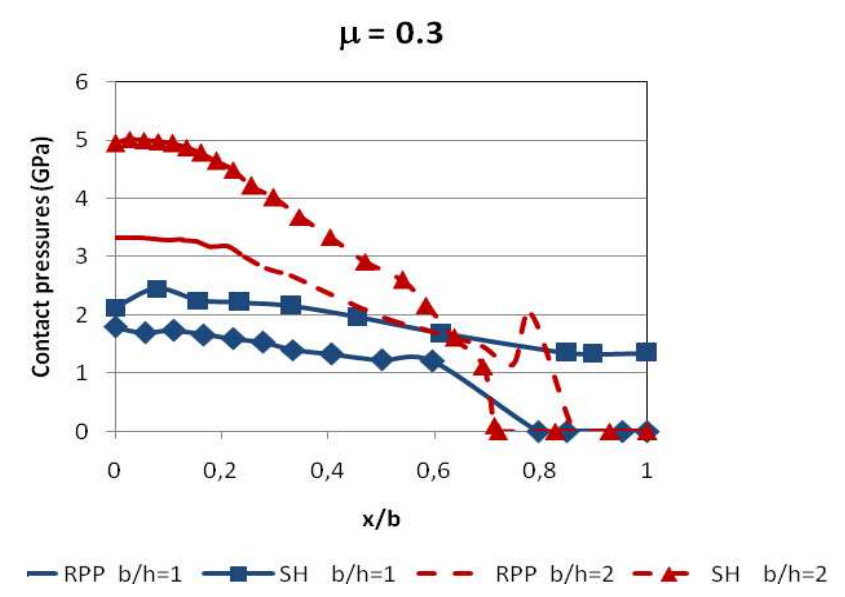

b)

Fig. 2. Contact pressure profiles considering two material models (Rigid Perfectly Plastic-RPP and Strain Hardened-SH) and two base to height ratios $(r=50 \%)$ : a) $\mu=0$ and b) $\mu=0.3$.

In the second case, when friction is considered (Fig. 2b), the results are quite different. The strain hardening has an important effect but also the $b / h$ ratio. Thus, the highest contact pressures are obtained when the $b / h$ ratio is higher. And the higher the $b / h$ ratio, the higher the differences obtained as a result of the strain hardening phenomenon. For example, the differences in contact pressures between RPP and SH materials are bigger for the highest $b / h$ ratio $(b / h=2)$.

Equivalent strains and stresses. Some other variables can be easily analyzed by using FE analysis. In Fig. 3 and 4 stress and strain diagrams can be observed in order to show these results in a intuitive way. In both figures, the equivalent stresses and strains are represented for $b / h=0.5, \mu=0.3$ and $r=$ $25 \%$, but in Fig. 3 the material is supposed to be a rigid perfectly plastic material and in Fig. 4 a strain hardened one. The influence of strain hardening on these results can be compared.
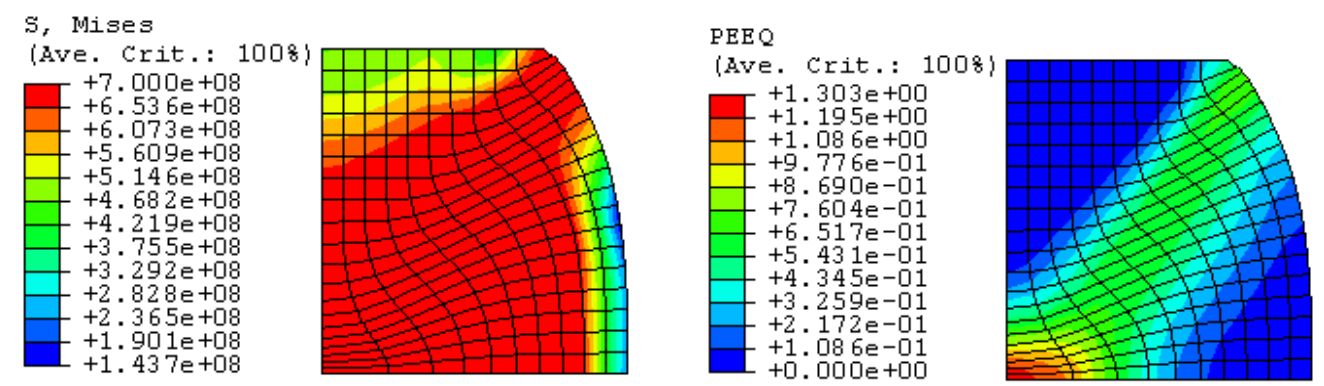

Fig. 3. Equivalent stress and strain diagrams for a RPP material $(b / h=0.5 ; \mu=0.3 ; r=25 \%)$ 

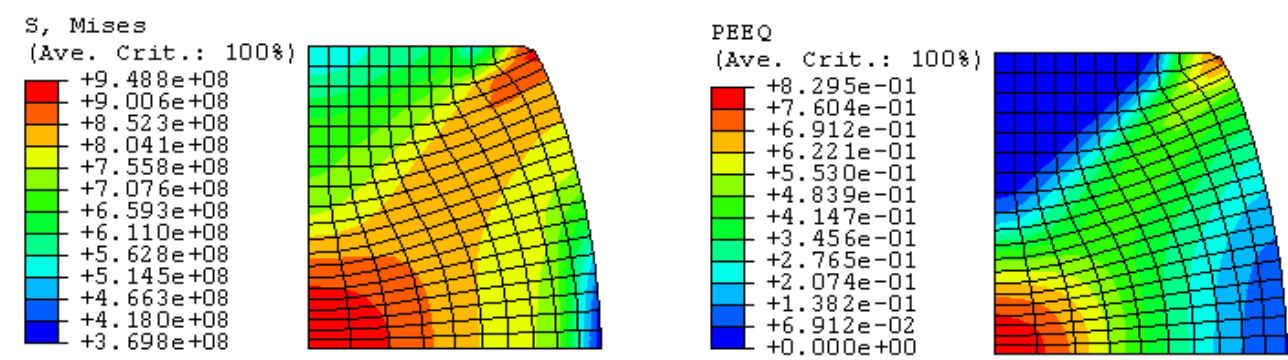

Fig. 4. Equivalent stress and strain diagrams for a SH material $(b / h=0.5 ; \mu=0.3 ; r=25 \%)$

\section{Conclusions}

In the last decades investigations in metal forming processes have been focused in the development of new processes that can overcome some of the limitations and/or disadvantages of conventional ones. Recently these studies are gaining in importance because of the increasing emergence of non conventional forging processes such as LIF processes. To improve their efficiency and the quality of the final products, and to fit the production requirements, it is advisable to get detail information about the conventional ones mainly through finite element models. With this purpose elementary processes of forging have been analyzed under plane strain conditions. Forces and contact pressure distributions have been obtained for different base to height ratios and several values of the friction coefficient; additionally, two models of material have been considered: a rigid perfectly plastic material and a strain hardened one. The results have shown that strain hardening has a high effect on the platen forces. When friction does not exist the geometry of the workpiece has not influence on forces. However, when friction is considered, the higher $b / h$, the higher the required force; the most important finding is that, for the same $b / h$ ratio, the effect of the strain hardening on the forces is higher when the friction conditions become more extreme. Contact pressure distributions have been analyzed for two cases: with and without friction. When friction is neglected the $b / h$ ratio has not influence on the contact pressures and the strain hardening has a small effect. In the case with friction, the $b / h$ ratio has an important influence on contact pressures, mainly for strain hardened materials. Furthermore, stress and strain distributions have been obtained in order to show their behavior under different conditions.

\section{Acknowledgements}

This work was financially supported by the Ministry of Economy and Competitiveness of Spain (Project DPI2009-07300) and the funds provided trough the Annual Grant Call of the E.T.S. Ingenieros Industriales (Industrial Engineering School) of UNED.

\section{References}

[1] J.M. Allwood, in: Proceedings of the 9th International Conference on Technology of Plasticity, edited by D.Y. Yang, Gyeonju (2008).

[2] A.M. Camacho, C. Vallellano, F.J. García-Lomas and M.A. Sebastián: Steel Res. Int. Vol. 81 (2010), p. 958.

[3] G.W. Rowe, C.E.N. Sturgess, P. Hartley and I. Pillinger: Finite-element plasticity and metalforming analysis (Cambrige University Press, USA 1991).

[4] F. Martin, L. Sevilla and C. Bermudo: Mater. Sci. Forum Vol. 713 (2012), p. 13.

[5] F.J. Olivares, A.M. Camacho and M.A. Sebastián: Adv. Mater. Research Vol. 498 (2012), p. 31.

[6] P. Hartley, C.E.N. Sturges and G.W. Rowe: Int. J. Mech. Sci. Vol. 22 (1980), p.743.

[7] D. Hibbitt, B. Karlsson and P. Sorensen, ABAQUS v6.11, User’s Manuals (2011). 